Revue Française de Civilisation Britannique

XVII-2 | 2012

Minorités, intégration en Grande-Bretagne et dans les pays du Commonwealth

\title{
La presse nationale britannique et le Mandat du Royaume-Uni en Palestine (1922-1939)
}

The British Mandate in Palestine as seen by the British Press (1917-1939)

Jean-Claude Sergeant

\section{(2) OpenEdition}

Journals

Édition électronique

URL : http://journals.openedition.org/rfcb/675

DOI : $10.4000 /$ rfcb. 675

ISSN : 2429-4373

Éditeur

CRECIB - Centre de recherche et d'études en civilisation britannique

Édition imprimée

Date de publication : 15 octobre 2012

Pagination : 81-102

ISBN : 2-911580-37-0

ISSN : 0248-9015

Référence électronique

Jean-Claude Sergeant, « La presse nationale britannique et le Mandat du Royaume-Uni en Palestine (1922-1939) », Revue Française de Civilisation Britannique [En ligne], XVII-2 | 2012, mis en ligne le 15 mars 2016, consulté le 19 avril 2019. URL : http://journals.openedition.org/rfcb/675 ; DOI : 10.4000/ rfcb. 675

Ce document a été généré automatiquement le 19 avril 2019

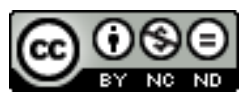

Revue française de civilisation britannique est mis à disposition selon les termes de la licence Creative Commons Attribution - Pas d'Utilisation Commerciale - Pas de Modification 4.0 International. 


\section{La presse nationale britannique et le Mandat du Royaume-Uni en Palestine (1922-1939)}

The British Mandate in Palestine as seen by the British Press (1917-1939)

Jean-Claude Sergeant

1 Puissance victorieuse au sortir de la Première Guerre mondiale, le Royaume-Uni avait obtenu à l'issue du conflit la reconnaissance d'une zone d'influence au Proche-Orient qui s'étendait de la Méditerranée au golfe persique, incluant la Palestine et la Mésopotamie. L'autorité du Royaume-Uni sur ces zones sera officiellement légitimée par l'approbation de mandats approuvés par la Société des Nations en 1922.

2 Cette date marque le début de la tutelle britannique, qui avait été précédée par une administration militaire installée en Palestine depuis l'entrée à Jérusalem du général Allenby en décembre 1917 après six mois de campagne contre les troupes turques. Le $1^{\mathrm{er}}$ juillet 1920, Herbert Samuel, haut-commissaire pour la Palestine, succédait à Louis Bols, dernier administrateur militaire de la région, qui exprimait, déjà, son inquiétude face à la remise en cause de ses pouvoirs dans une lettre adressée à l'état-major britannique au Caire: "Mon autorité et celle de chaque département de mon administration est contestée ou contrecarrée par la commission sioniste et je suis convaincu que cette situation ne peut se prolonger sans mettre gravement en péril la paix publique ${ }^{1}$. »

Entre-temps, la Déclaration Balfour du 2 novembre 1917 avait exprimé le soutien du gouvernement britannique à la création en Palestine d'un « foyer national pour le peuple juif ", déclaration qui sera intégrée au texte définissant les termes du mandat en Palestine confié au Royaume-Uni par la Société des Nations. Vingt ans plus tard, le gouvernement britannique en arrivait à la conclusion que seul le partage de la Palestine en deux États autonomes pouvait permettre de régler le problème palestinien auquel une douzaine de rapports de commissions diverses avait été consacrés au cours de la période, sans parvenir à trouver une solution permettant aux communautés arabe et juive de vivre en bonne intelligence dans la région que les Britanniques s'étaient attribuée dans le cadre de 
l'accord Sykes-Picot signé en 1916, tenu secret jusqu'à ce que les autorités bolchéviques le rendent public. En contrepoint de la Déclaration Balfour, le rapport de la Commission royale publié en 1937 - le rapport Peel - prenait acte de l'impasse dans laquelle se trouvaient les Britanniques :

Sous la pression de la guerre mondiale, le gouvernement britannique a fait des promesses aux Arabes et aux Juifs afin d'obtenir leur soutien, sur la base desquelles les deux communautés ont entretenu certains espoirs [...]. Un conflit insoluble est né entre les deux communautés nationales dans ce territoire exigu [...]. Ce conflit était en germe dès le départ. ${ }^{2}$

4 Il importera, dans un premier temps, de retracer l'évolution de la position de différents gouvernements britanniques qui se sont succédé sur la question palestinienne et d'en rappeler les principaux jalons, avant d'aborder l'examen du positionnement de la presse face à cet enjeu rémanent sur lequel quelques propriétaires de journaux ont tenté de peser.

\section{Principes et ajustements de la politique britannique en Palestine}

5 L'implication du Royaume-Uni en Palestine, consacrée par l'attribution d'un mandat de la SDN en juillet 1922, avait été préparée par l'intérêt que portait une partie de la classe politique britannique à la situation des Juifs en Europe orientale. Chaïm Weizmann, président de l'Organisation sioniste mondiale, rappelle dans un discours au dix-septième Congrès sioniste (1931) la protection que les consuls britanniques en Palestine accordaient aux Juifs avant 1914. Il évoque également le projet de création d'un foyer national juif en Afrique orientale débattu à la Chambre des communes en 1904, à l'initiative de Joseph Chamberlain, alors ministre des Colonies (Colonial Office). Selon Weizmann, la Déclaration Balfour qui, en 1917, ouvrait de nouvelles perspectives aux communautés juives d'Europe centrale et orientale, s'expliquait par trois facteurs. Le premier, d'ordre sentimental, était le fruit de la familiarité du peuple britannique, formé à la culture biblique, avec l'attachement des communautés juives à la terre de leurs ancêtres. Le second facteur mis en avant par Weizmann concernait l'idéal démocratique qui prenait alors forme et auquel le Président Wilson allait donner corps avec sa proclamation des Quatorze Points (janvier 1918). Pour que l'acte de naissance de ce nouveau monde en gestation soit totalement vierge, il convenait de régler l'ancien contentieux avec le peuple juif ${ }^{3}$.

6 La dernière considération avancée par Weizmann, d'ordre stratégique celle-là, était en fait double. Il s'agissait d'une part pour le gouvernement britannique de s'assurer le soutien des Juifs américains afin qu'ils fassent pression sur le Congrès pour qu'il autorise l'entrée en guerre des États-Unis. D'autre part, la perspective de l'implantation d'un point d'appui en Palestine permettant de protéger le canal de Suez et donc les voies de communication avec l'Inde plaidait en faveur de l'établissement d'un État juif entretenant d'étroites relations avec Londres. En novembre 1914, Herbert Samuel, membre du gouvernement Asquith et futur haut-commissaire en Palestine, avait évoqué cette option avec le Secrétaire au Foreign Office, Edward Grey, qui semble avoir été convaincu par le projet $^{4}$. Lloyd George, lui-même, avait marqué son intérêt pour le projet, non pas tant par sympathie pour la cause sioniste, que par souci de faire pièce aux ambitions françaises dans la zone. Selon Weizmann, cette dernière considération, qu'il qualifie d'utilitaire, 
était loin d'être centrale, et il en veut pour preuve la prudence des Britanniques qui, lors des débats à la SDN concernant le choix de la puissance mandataire en Palestine, auraient volontiers accepté de n'être que l'un des partenaires d'un condominium occidental.

7 De la Déclaration Balfour - cette "déclaration de sympathie pour les aspirations sionistes » selon les termes de la lettre adressée par Arthur Balfour à lord Rothschild ${ }^{5}$ allait découler une série de recadrages et de redéfinitions des conditions d'application du principe central - « l'établissement en Palestine d'un foyer national pour le peuple juif »à mesure que s'intensifieront les antagonismes entre les deux communautés et la défiance à l'égard de la puissance de tutelle.

8 La première clarification est intervenue sous la forme d'un Livre blanc ${ }^{6}$ publié en 1922, quelques mois avant la confirmation du mandat britannique par la SDN et deux ans après l'arrivée à Jérusalem d'Herbert Samuel en qualité de haut-commissaire. La nomination d'un sioniste déclaré à la tête de l'administration britannique en Palestine avait suscité des manifestations hostiles parmi la communauté arabe qui, l'année suivante, considérera comme de très mauvais augure le transfert de la gestion politique du mandat du Foreign Office au Département Moyen-Orient créé en février 1921 au sein du Colonial office dirigé par Winston Churchill qui ne faisait pas mystère de son soutien à la cause sioniste. Le recrutement par le Département du colonel Richard Meinertzhagen, pro-sioniste ardent, en qualité d'expert militaire, ne pouvait qu'accroître les appréhensions de la communauté arabe qui constituait à l'époque $90 \%$ de la population palestinienne.

Les violences perpétrées contre les Juifs en 1920 et en mai 1921 incitèrent Samuel à décréter la suspension provisoire de l'immigration juive en Palestine. Dans une correspondance à Churchill, le haut-commissaire ne cachait pas son inquiétude : «Je ne peux m'ôter de l'esprit la possibilité de voir ces perturbations se renouveler ou même, si j'en crois les avertissements de mes conseillers militaires, d'assister à un soulèvement général ${ }^{7}$. " Dix jours auparavant (3 juin 1921), Herbert Samuel tentait d'apaiser les arabes en s'efforçant de clarifier le «regrettable malentendu » qu'avait suscité la Déclaration Balfour en assurant musulmans et chrétiens de Palestine que «le gouvernement britannique, qui avait reçu mandat d'assurer le bonheur des populations de Palestine, ne leur imposerait jamais une politique qu'elles considèreraient comme contraire à leurs intérêts religieux, politiques et économiques ${ }^{8}$.»

C'est dans ce contexte que Winston Churchill et ses conseillers ont élaboré, en étroite concertation avec Herbert Samuel, le premier Livre blanc proposant une actualisation de la politique en Palestine, en tentant de trouver un point d'équilibre entre l'engagement en faveur de l'établissement du foyer national promis aux Juifs et le souci de répondre aux inquiétudes des populations musulmane et chrétienne. Il s'agissait, déjà, de clarifier la portée du concept ambigu de « foyer national pour le peuple juif » et de réaffirmer que la présence des Juifs en Palestine était de droit et non pas tolérée - « of right and not on sufferance ». Par ailleurs, le Livre blanc indiquait que la mise en œuvre de la Déclaration de 1917 impliquait le développement du peuplement juif par l'apport d'immigrants, cette immigration devant toutefois être régulée en fonction des capacités d'absorption de l'économie du pays. Le document précisait également, pour lever les craintes de la communauté arabe, que les immigrants juifs ne devaient pas constituer une charge supplémentaire pour les Palestiniens - «the people of Palestine » - et que leur présence ne devait en aucun cas priver cette communauté de ses emplois. Enfin, le Livre blanc annonçait la création d'un Conseil législatif composé de dix représentants élus et de dix membres nommés, premier pas vers une future autonomisation du territoire. 
11 Rédigé dans une perspective de clarification du projet politique du gouvernement en Palestine en vue de la confirmation du mandat par le Conseil de la SDN appelé à se prononcer le 24 juillet 1922, le document présenté par Churchill ne parvenait pourtant pas à faire l'unanimité. Le 21 juin, la Chambre des lords adoptait par soixante voix contre vingt-neuf une motion proposée par lord Islington s'opposant à l'attribution du mandat au Royaume-Uni, au motif que celui-ci était en contradiction avec l'engagement pris en 1915 à l'égard des Arabes ${ }^{9}$ et avec « les sentiments et les souhaits de la grand majorité de la population de Palestine». Après le rejet d'une motion identique par les Communes, le gouvernement put préparer les élections au Conseil législatif palestinien auxquelles il dut au bout du compte renoncer suite à l'appel au boycott lancé par les organisations arabes. Le projet de création d'une Agence arabe faisant pendant à l'Agence juive qui assistait le Conseil exécutif à Jérusalem se heurta également à une fin de non recevoir, si bien que l'Administration britannique en Palestine en fut réduite à mettre en place une instance de représentation de la population arabe, le Conseil supérieur musulman, présidé par le Grand Mufti de Jérusalem, à laquelle sera transféré le contrôle des organes de financement et des tribunaux civils musulmans. Présentée par Herbert Samuel comme la mise en place d'un intermédiaire entre les musulmans et les autorités britanniques, la création du conseil marquait en fait une nouvelle étape dans le dessaisissement de l'Administration britannique de ses pouvoirs. S'agissant de la communauté juive, ceux-ci avaient déjà été largement délégués à la Commission sioniste - l'organe exécutif sioniste dont le rôle d'auxiliaire de l'Administration avait été explicitement reconnu par l'article 4 du mandat. Incapable de mettre en place une structure législative représentative et unitaire, le gouvernement britannique, en l'occurrence le haut-commissaire et le Colonial Office, avait ainsi été contraint d'accepter un partage du pouvoir avec deux organes distincts au service des seuls intérêts des communautés qu'ils géraient de façon quasi autonome.

12 En 1930, le gouvernement britannique tentait une nouvelle fois de "dissiper les malentendus existants et de définir plus précisément ses intentions » en publiant un nouveau Livre blanc ${ }^{10}$ rédigé sous la responsabilité de Sidney Webb - lord Passfield nommé ministre en charge du Colonial office dans le gouvernement de Ramsay MacDonald issu des élections de 1929. Cette nouvelle tentative de refondation de la politique britannique en Palestine s'imposait après les violentes émeutes qui avaient fait plus de deux cents morts dans différentes villes du territoire en août 1929. Le Colonial office avait dépêché une commission d'enquête sur place dirigée par un ancien magistrat colonial, Walter Shaw, qui remit son rapport en mars 1930. Ses principales recommandations concernaient la protection des droits des communautés non juives, notamment en matière d'accès à la propriété des terres agricoles et l'application plus stricte des dispositions régissant l'immigration. Le rapport préconisait également que soient plus précisément définies les limites des prérogatives de l'Organisation sioniste dans la gestion des affaires palestiniennes.

Ce rapport marquait une nette réorientation des priorités britanniques en faveur des doléances de la majorité musulmane. Une commission supplémentaire, dirigée par John Hope Simpson, fut chargée d'apporter un complément d'information afin de nourrir la nouvelle doctrine palestinienne du gouvernement. Telle qu'elle était exposée dans le Livre blanc de lord Passfield, cette doctrine visait à mettre résolument les deux communautés en face de leurs responsabilités. Le document annonçait le renforcement des forces de sécurité à la disposition des autorités de Jérusalem; deux escadrons de 
l'Armée de l'air et quatre sections d'unités blindées viendraient appuyer les deux bataillons d'infanterie déjà sur place. Plus fondamentalement, le Livre blanc, reprenant les points principaux du Livre blanc de 1922 et les articles pertinents du mandat, réaffirmait l'intention du gouvernement de procéder une nouvelle fois à la mise en place d'un conseil législatif, mais c'est surtout sur les questions de développement économique et social et sur le problème de l'immigration juive que portait l'essentiel du document. La politique d'acquisition foncière appliquée par l'Organisation sioniste aboutissait à déposséder de leurs terres les paysans arabes qui se trouvaient, par ailleurs, exclus du marché du travail du fait de la pratique qui empêchait les propriétaires juifs d'avoir recours à une main-d'œuvre non juive. La politique d'immigration était plus directement mise en cause par les concepteurs du Livre blanc qui précisaient :

Si l'immigration juive a pour effet d'empêcher la population arabe d'obtenir les emplois qui lui permettraient de suffire à ses besoins ou si le chômage parmi la communauté juive a un effet négatif sur le marché du travail dans son ensemble, il appartient à la puissance mandataire, selon les termes du mandat, de réduire et, le cas échéant, de suspendre cette immigration tant que les chômeurs des «autres sections » n'auront pas retrouvé un emploi ${ }^{11}$.

Les organisations sionistes considérèrent ce document comme une provocation et un reniement de l'engagement de 1917. Selon un universitaire travaillant dans une perspective sioniste : «Le Livre blanc de lord Passfield a été interprété, du moins par les Juifs, comme un document délibérément rédigé pour humilier, ce que d'ailleurs confirmait Beatrice Webb, peu suspecte de sympathie sioniste [...], qui notait dans son Journal [le 26 octobre 1930] : “cet exposé [...] est un document mal rédigé et dépourvu de $\operatorname{tact}^{12}$." »

En signe de protestation, Chaïm Weizmann se démit de ses fonctions à la tête de l'Agence juive, tandis que des manifestations à Londres, New York, Varsovie et en Afrique du Sud dénonçaient le « reniement » britannique et que les ténors conservateurs et libéraux Stanley Baldwin, Leo Amery, Austen Chamberlain et Lloyd George en tête - exprimaient dans la presse leur désaccord. Faute d'obtenir du gouvernement la mise en chantier d'un autre Livre blanc rectificatif, les dirigeants sionistes obtenaient du Premier ministre MacDonald la mise en place d'un sous-comité du Cabinet, sous la présidence du ministre des Affaires étrangères, Arthur Henderson, chargé de faire le point, en concertation avec les représentants sionistes, sur les priorités britanniques en Palestine, ce qui revenait à désavouer lord Passfield et à remettre en cause les recommandations de sa commission.

Les travaux du sous-comité aboutirent à un projet de lettre qui, remanié à cinq reprises, fut soumis à MacDonald. Il était prévu que cette lettre soit adressée à Chaïm Weizmann après que lecture en aurait été donnée aux Communes, de façon à ce qu'elle soit consignée au Hansard, le procès-verbal officiel des débats parlementaires. Le Times publiera le 15 février 1931 le texte de cette longue mise au point de dix-huit paragraphes dont l'introduction donnait toute satisfaction aux dirigeants sionistes :

Afin de dissiper certaines interprétations erronées et certains malentendus relatifs à la politique du gouvernement de Sa Majesté concernant la Palestine, telle qu'elle a été exposée dans le Livre blanc d'octobre 1930, et qui ont fait l'objet d'un débat à la Chambre des communes, afin, également, de répondre à certaines critiques formulées par l'Agence juive, j'ai le plaisir de vous adresser le texte qui suit exposant notre position, qu"il conviendra de considérer comme l'interprétation officielle du Livre blanc concernant les points abordés dans la présente lettre ${ }^{13}$. 

d'acquisitions foncières permirent aux Juifs de Palestine de consolider le foyer national, ce dont convenait Weizmann dans ses Mémoires: "C'est à la lettre que MacDonald m'a adressée que l'on doit le changement qui s'est opéré dans l'attitude du gouvernement et de l'Administration en Palestine, changement qui nous a permis d'accomplir les remarquables progrès des années suivantes ${ }^{14}$.» 1936, après que la France eut accepté de mettre un terme à son mandat en Syrie et que Londres eut jugé préférable de replier ses troupes en Égypte sur la zone du canal de Suez. Par ailleurs, l'Irak avait vu sa souveraineté reconnue en 1932, tandis que la Transjordanie s'acheminait vers une autonomie élargie. Les Arabes de Palestine n'avaient pas oublié que l'esprit du mandat impliquait, à terme, le passage à la pleine souveraineté du territoire temporairement placé sous tutelle et c'est autour de cette revendication que se cristallisera en 1936 l'opposition arabe à l'ordre britannique incarné par les forces de sécurité - 20000 hommes à cette époque contre 5000 en 1920 -, accompagnée de violences et d'attentats contre les Juifs de Palestine. En avril 1936, le territoire fut largement paralysé par une grève générale organisée par un collectif d'opposants arabes dirigé par le Grand Mufti de Jérusalem, qui ne prendra fin qu'en prévision de l'arrivée en Palestine des six membres d'une nouvelle commission royale chargée de faire la lumière sur les causes des "perturbations»- le terme original alors en vigueur était " disturbances » - et de proposer une issue à ce problème qui s'enkystait depuis vingt ans.

Présidée par lord Peel, ancien ministre chargé des affaires indiennes, la commission fut accueillie à Jérusalem en novembre 1936. Elle procéda en Palestine à de multiples auditions et à de nombreuses visites sur le terrain avant de regagner Londres en février 1937 où elle compléta son information, notamment auprès de la hiérarchie militaire. C'est en juillet que fut publié son rapport ${ }^{15}$ qui, de l'avis d'une spécialiste, « constitue l'analyse la plus complète et la plus extensive de la situation en Palestine jamais produite par une instance officielle britannique ${ }^{16}$." L'essentiel des quatre cents pages du rapport retrace l'historicité des peuplements juif et arabe en Palestine et fait l'inventaire du contentieux entre les deux communautés. Les membres de la commission se sont divisés sur la question de savoir s'il fallait suspendre provisoirement l'immigration juive ou bien considérer que l'établissement du foyer national promis en 1917 étant réalisé, il convenait d'y mettre définitivement un terme. La commission a préféré trancher radicalement en concluant que l'idéal qui sous-tendait le mandat, à savoir l'émergence progressive d'une identité nationale palestinienne, s'étant avéré inaccessible, il restait à en tirer la conclusion. Celle-ci, détaillée dans les vingt dernières pages du rapport, préconisait le partage du territoire en trois zones, dont deux auraient vocation à se muer en États, l'un arabe, l'autre juif, la troisième zone incluant Jérusalem et ses lieux saints ainsi qu'un certain nombre de villes dont le port d'Haiffa restant sous contrôle britannique. Conscients que cette solution chirurgicale ne serait pas du goût des Juifs, pas plus que de celui des Arabes de Palestine, la commission concluait son rapport par ce proverbe: «Half a loaf is better than no bread». Visiblement décontenancé par les propositions de la commission, le gouvernement n'avait d'autre recours que d'en nommer une nouvelle - The Woodward Commission - afin d'en évaluer les modalités pratiques d'application, avant de conclure en novembre 1938 que «la proposition de créer en Palestine des États juif et arabe indépendants comportait des difficultés politiques, administratives et financières telles que cette solution [était] inapplicable ${ }^{17}$.»

Revue Française de Civilisation Britannique, XVII-2 | 2012 
20 Le gouvernement de Neville Chamberlain ne pouvait pourtant pas laisser la question palestinienne en l'état. Dans la perspective de plus en plus vraisemblable de l'ouverture d'un conflit avec l'Allemagne et l'Italie, il importait de s'assurer du soutien ou, à tout le moins, de la neutralité des pays arabes. C'est dans ce contexte que s'est ouverte le 7 février 1939 à Londres une série de "conférences » organisées par le Colonial Office, réunissant des représentants de l'Agence juive, des Arabes de Palestine ainsi que des envoyés des États arabes voisins, la négociation étant conduite avec chaque délégation séparément. Le Colonial Office avait fait savoir que faute de pouvoir parvenir à un accord entre les différentes parties, le gouvernement serait amené à produire ses propres conclusions. Le 17 mars, les participants se séparaient sur un constat d'échec, laissant au Colonial office le soin d'élaborer un nouveau plan pour la Palestine. Ce sera l'objet du dernier Livre blanc sur la question, publié le 17 mai $1939^{18}$. Le document abordait les trois sujets litigieux traditionnels: l'enjeu constitutionnel, l'immigration et la question des terres. Sur le premier thème, le gouvernement envisageait la création d'un État palestinien indépendant dont le gouvernement serait partagé entre Juifs et Arabes de telle sorte que les intérêts de l'une et l'autre communauté soient préservés. Cette structure de gouvernement serait mise en place au cours d'une période de dix ans une fois la paix revenue et l'ordre rétabli en Palestine. Les responsables britanniques prévoyaient en outre de limiter à 75000 le nombre d'immigrants juifs admis en Palestine au cours des cinq années suivant l'adoption du Livre blanc, toute immigration supplémentaire étant soumise ensuite à l'accord des Arabes de Palestine. Enfin, s'agissant de la question foncière, le document rappelait que le transfert de terres entre Arabes et Juifs n'avait fait l'objet d'aucune restriction mais qu'afin de prévenir l'augmentation d'une population agricole arabe privée de terres, il convenait de doter le hautcommissaire du pouvoir de contrôle et d'arbitrage en matière de transferts fonciers.

21 Les Arabes n'ont pas tardé à rejeter les projets britanniques, au motif qu'ils ne prévoyaient pas l'arrêt immédiat de l'immigration juive, l'Agence juive, de son côté, dénonçant cette trahison - «breach of faith » - qui aboutirait «à parquer les Juifs dans un ghetto territorial dans leur propre patrie ${ }^{19}$.» Soumis pour approbation à la Commission des mandats de la SDN, conformément à la procédure, le projet britannique était jugé non conforme aux termes du mandat confié au Royaume-Uni, mais la déclaration de guerre, intervenue le 3 septembre 1939, allait laisser la question pendante jusqu'à la fin des hostilités.

\section{La presse britannique entre indifférence et interventionnisme}

La couverture de la question palestinienne par la presse britannique, entendue ici au sens restrictif de presse nationale ou quasi nationale - on songe au Manchester Guardian s'appréhende sous plusieurs aspects, celui d'abord de l'enregistrement des événements, celui du commentaire ensuite et enfin sous l'aspect tribunitiel permettant l'exposition dans le débat public de points de vue et de mises au point dans l'espace réservé au courrier des lecteurs (Letters to the Editor) dans les principaux titres et, en premier lieu dans le Times. Sans qu'il soit possible de mesurer l'impact de la couverture de la question par la presse sur les décisions politiques et encore moins sur l'opinion publique à une époque où les sondages d'opinion commençaient à peine à poindre, on peut néanmoins tenir pour acquis que les commentaires de la presse étaient pris en compte par les 
différents protagonistes $\mathrm{du}$ problème. Ainsi, Chaïm Weizmann, président de l'Organisation sioniste, déclarait en 1922 dans un discours public à Oxford :

Le lecteur de journaux type pense qu'il existe en Palestine un État juif, entendant par « État » ce que ce terme désigne quand on songe à ce que fut la Prusse, c'est-àdire une sorte d'entité qui se soucie comme d'une guigne de quiconque n'appartient pas à l'élite dominante. Il estime que cet État - cela a été dit - est financé grâce à la générosité britannique et alimenté par les impôts des Britanniques. Telle est, plus ou moins, l'opinion qui prévaut dans l'esprit du lecteur de journaux moyen ${ }^{20}$. époque, la presse britannique s'activait contre le sionisme et singulièrement contre Weizmann, accusés d'être responsables des difficultés dans laquelle se débattait la Grande-Bretagne en Palestine, aux frais du contribuable britannique ${ }^{21}$.» C'est précisément la thématique que développa, de façon particulièrement vigoureuse, le Daily Mail jusqu'à la mort de son fondateur, lord Northcliffe, en 1922 et qui fut exploitée, sous une forme plus nuancée, après le départ en 1926 du directeur de rédaction, Thomas Marlowe, que Northcliffe avait recruté en 1899, trois ans après le lancement du quotidien.

\section{Situation de la presse dans l'Entre-deux-guerres}

La presse nationale a connu une certaine stabilité au cours des vingt années qui ont séparé les deux conflits, du moins si l'on s'en tient au nombre de titres quotidiens disponibles. En 1919, le lecteur londonien pouvait choisir entre onze quotidiens du matin et six quotidiens du soir. A la veille de la Seconde Guerre mondiale, le choix était pratiquement aussi large. Certes, trois titres avaient été absorbés par d'autres publications, le Morning Post étant intégré au Daily Telegraph, tandis que la Westminster Gazette fusionnait avec le Daily News, avant que ne s'opère la jonction avec le Daily Chronicle pour donner naissance en 1930 au News Chronicle, organe du parti libéral. Ces trois disparitions étaient compensées par le lancement en 1930 du Daily Worker, quotidien officiel du parti communiste, et par la refondation du Daily Herald qui, passé sous le contrôle de la confédération syndicale (TUC) en 1922, avait évité la faillite grâce à l'intervention de la société Odhams Press, qui propulsera le Daily Herald en tête des ventes des quotidiens - plus de deux millions d'exemplaires au milieu des années trente - au moyen de stratégies commerciales offensives. La presse du soir, qui comptait encore six titres en 1919, résista en revanche moins bien à l'évolution des comportements du public, sollicité par de nouvelles formes de loisir, la radiodiffusion et le cinématographe. Les influentes publications qui alimentaient les conversations dans les clubs - la Westminster Gazette, devenue quotidien du matin avant son absorption par le Daily News, la Pall Mall Gazette et The Globe - disparaissaient, sans successeurs, d'un marché que se disputeront dorénavant trois titres - The Star, The Evening News et The Evening Standard - jusque dans les années 1960.

Cette apparente stabilité dissimulait en fait une importante réorganisation des forces capitalistes qui dominaient le marché. Le démembrement de l'empire Northcliffe qui, en 1915, incluait les titres phares de la presse nationale - le Times, le Daily Mail, l'Observer, l' Evening News et la Weekly Dispatch-, sans compter les titres régionaux, amena une recomposition du secteur. Le Times sera vendu à la famille Astor qui avait déjà acquis l' Observer avant la mort de Northcliffe. Harold Harmsworth (lord Rothermere), frère de lord Northcliffe, à qui avait été confiée la gestion du Daily Mirror, fondé par Northcliffe en 
1903, mit en vente ses parts sur le marché, ce qui permit la constitution d'une nouvelle société, Daily Mirror Newspapers Ltd, dirigée par Cecil King qui fit de ce titre le fleuron de la presse populaire aux cours des années 1940. La période de l'Entre-deux-guerres sera également marquée par l'exacerbation de la concurrence entre les principaux quotidiens populaires, parmi lesquels le Daily Express n'était pas le moins agressif. Racheté par Max Aitken, futur lord Beaverbrook, en 1916, le quotidien, renforcé par le dominical Sunday Express fondé en 1918, n'allait pas tarder à faire puissamment résonner la voix de son propriétaire très impliqué dans les affaires politiques de sa patrie d'adoption.

Les bouleversements qui affectaient périodiquement l'équilibre du secteur de la presse acquisitions, fusions, disparitions - étaient suivis avec attention par les rédactions qui ne manquaient pas de commenter les péripéties en cours. Ainsi, le 2 juin 1930, le Daily Express annonçait en "une» le rachat du Daily Chronicle par le Daily News sous le titre «La Tragédie du 'Daily Chronicle'». Cette "tragédie» s'expliquait, selon le quotidien, par l'instabilité de la structure de gestion du journal, lequel avait connu trois propriétaires différents depuis la guerre, instabilité dont le rédacteur soulignait implicitement le contraste avec l'engagement financier sans faille de Beaverbrook en faveur de ses journaux. L'auteur de l'article du Daily Express voyait, en outre, dans la disparition du quotidien libéral la fin de l'époque où les journaux étaient liés à un parti politique - «tied party organs » - à laquelle avait succédé l'ère des journaux indépendants, commentaire pour le moins paradoxal au moment où Beaverbrook mettaient ses journaux au service de l'United Empire Party qu'il avait fondé pour s'opposer à la politique de libre échange défendue par le parti conservateur.

\section{Les différentes formes d'intervention de la presse dans la question palestinienne}

27 L'implication de la presse dans l'évolution de la politique du gouvernement britannique en Palestine reste limitée, même si, au début des années 1920, elle a pu colorer la perception qu'en avait le public. Cette implication était naturellement fonction de l'intérêt que lui portaient les éditeurs de journaux et les directeurs de rédaction. À cet égard, on peut opposer le soutien régulier, mais sobre, à la cause sioniste apporté par le Manchester Guardian à l'hostilité bruyante déployée par lord Northcliffe.

C'est à C.P. Scott, propriétaire et directeur de la rédaction du Manchester Guardian jusqu'en 1929, que Chaïm Weizmann doit ses premiers contacts avec les responsables politiques britanniques. Né en Russie, chimiste de formation, Weizmann avait été recruté en 1904 par l'université de Manchester où ses travaux sur l'acétone avaient retenu l'attention des militaires. En 1916, Weizmann était en charge d'un programme d'extraction de la nitrocellulose du maïs en vue de la production de cordite, un puissant explosif. Le responsable sioniste avait eu l'occasion de rencontrer Balfour à Manchester en 1906, mais ce n'est qu'en 1914 qu'il fut reçu par Lloyd George sur la recommandation de Scott qui le mit également en contact avec Herbert Samuel. En juin 1917, Balfour le recevait en compagnie de lord Rothschild et c'est au cours de cet entretien que le ministre des Affaires étrangères leur promit « un document dans lequel le gouvernement britannique exprimerait sa sympathie avec le mouvement sioniste et son intention de soutenir la création d'un foyer national juif en Palestine ${ }^{22}$.» C.P. Scott n'était pas sioniste. Le quotidien s'était peu intéressé à la Palestine entre 1914 et 1916, ce qui n'empêcha pas 
Scott de saluer la Déclaration Balfour en ces termes : "Nous parlons de la Palestine comme d'un pays, mais ce n'est pas un pays. C'est, à présent, à peine plus qu'un petit district dans le vaste empire ottoman tyrannique. Mais elle deviendra un pays ; ce sera celui des Juifs $\mathrm{s}^{23}$."

Si Scott s'était intellectuellement rallié à la cause défendue par Weizmann, certains de ses collaborateurs étaient totalement acquis au mouvement sioniste. Spécialiste des questions militaires, Herbert Sidebotham était arrivé au sionisme par l'analyse stratégique. Il avait très tôt défendu l'idée de faire de la Palestine un État-tampon lié au Royaume-Uni, destiné à protéger le canal de Suez. Il concluait un éditorial publié le 22 novembre 1915 par la recommandation suivante : «La Palestine doit soit être intégrée à l'Égypte [...] soit être un Etat-tampon incapable de faire preuve d'hostilité envers l'Egypte. C'est de cette condition que dépend l'avenir de l'empire britannique en tant que puissance impériale maritime ${ }^{24}$." Au sein de la rédaction du Manchester Guardian, Sidebotham côtoyait un autre éditorialiste, Harry Sacher, diplômé d'Oxford et beau-frère de Simon Marks, le fils du fondateur des Marks and Spencer's Penny Bazaars, qui entretenait des relations amicales avec Chaïm Weizmann lors de son séjour à Manchester. Tout au long de la période, le Manchester Guardian s'efforcera de maintenir une ligne rédactionnelle équilibrée, ne remettant cependant pas en cause son soutien au foyer national promis par Balfour.

Il n'en allait pas de même des autres organes de Fleet Street. Lord Beaverbrook est taxé d'antisionisme par Jason Tomes ${ }^{25}$, mais son biographe, A.J.P. Taylor, l'exonère de tout soupçon d'antisémitisme. «Beaverbrook n'avait aucune sympathie pour l'antisémitisme ", note-t-il, après avoir néanmoins fait état d'une lettre en date du 9 décembre 1938 accusant les Juifs d'être à l'origine des risques de guerre contre lesquels le gouvernement britannique tentait par tous les moyens de se prémunir : «Voilà des années que je prédis qu'il n'y aura pas de guerre. Mais, au bout du compte, je suis ébranlé. Les Juifs peuvent nous mener à la guerre, non pas consciemment, tel n'est pas leur projet, mais ils nous conduisent inconsciemment vers la guerre. Leur influence politique nous conduit dans cette direction $^{26}$. Et Beaverbrook de procéder à un inventaire de cette influence dans la presse nationale :

Les Juifs sont très solidement implantés dans la presse de ce pays. J'estime qu'un tiers des lecteurs du Daily Telegraph est juif. Il n'est pas impossible que le Daily Mirror soit contrôlé par des Juifs. Les propriétaires du Daily Herald sont juifs. Quant au News Chronicle, il devrait s'appeler le Jewish Chronicle, non pas tant à cause de ses propriétaires qu'à cause de ses sympathies. Il ne reste en fait que le Times, le Daily Mail et l'Express et encore, je ne suis pas sûr du Daily Mail ${ }^{27}$."

31 Cette lettre, que Taylor qualifie de "déplorable ", révèle l'ambiguïté du personnage qui, tout en laissant paraître dans l'Evening Standard qu'il contrôlait un compte rendu très favorable du livre d'Hilaire Belloc The Jews, peu de temps après qu'un article du Sunday Express eut expliqué, sous le titre "The Jews », que la montée de l'hostilité envers les Juifs n'avait d'autre cause que le sionisme, informe en 1927 lord Rothermere qu'il a interdit à ses journalistes de mentionner Hilaire Belloc et G.K. Chesterton dans les colonnes de ses journaux.

Beaverbrook justifie son antisionisme par l'entretien qu'il avait eu en 1917 avec deux parlementaires juifs antisionistes, Lionel de Rothschild et Charles Henry, qui lui avaient fait part des réserves qu'avait exprimées Lloyd George, alors Premier ministre, à l'égard du projet d'établissement d'un foyer national juif en Palestine. Beaverbrook était à cette époque secrétaire d'État responsable de l'information et de la propagande. À ce titre, il 
était notamment chargé de diffuser aux États-Unis la position britannique sur la question palestinienne. À la suite de cet entretien, Beaverbrook avait largement atténué la coloration sioniste des messages diffusés par ses services, s'attirant ainsi un sec rappel à l'ordre d'Arthur Balfour, en charge des Affaires étrangères : «La politique du gouvernement de Sa Majesté en Palestine est celle définie par le ministre des Affaires étrangères dans son dernier discours. Tant qu'elle n'aura pas été modifiée officiellement, elle ne saurait en aucune façon être affectée par des conversations entre Sir Charles Henry et le Premier ministre ${ }^{28}$."

Au printemps de 1923, accompagné d'un journaliste du Sunday Express, Beaverbrook se rendit en Palestine pour se forger sa propre opinion, ainsi qu'il le déclare à son entourage. Il adresse à la rédaction du Daily Express au cours du mois d'avril une série de câbles centrée sur ce qu'il percevait comme l'échec programmé du pari sioniste. Mais la Palestine ne fut qu'occasionnellement un thème de préoccupation majeur pour Beaverbrook, de plus en plus engagé dans son combat en faveur du système de préférence impériale et dans son action d'opposant au courant belliciste qui montait en puissance face à l'arrivée au pouvoir des totalitarismes en Europe. À titre d'exemple, l'édition du 14 février 1931 du quotidien se bornait à reproduire en page deux un bref extrait de la lettre de Ramsay MacDonald à Chaïm Weizmann, assorti de la «titraille " suivante: " Premier's Palestine Peace Gesture / 'We adhere to Mandate' / Zionist leader Gratified by Statement / Revival of Confidence (Dr Weizmann ». Aucun commentaire éditorial ne venait en revanche éclairer cet important désaveu du Livre blanc de 1930.

Lord Northcliffe s'est lui aussi rendu en Palestine, un an avant Beaverbrook, au cours d'un voyage autour du monde entrepris à la fin de sa vie. Voici le commentaire qu'inspirait cette brève étape à Norman Bentwich, Attorney General (conseiller juridique) du gouvernement de Palestine de 1922 et 1931 :

La campagne de désinformation menée par la Presse et au Parlement a connu son apogée au cours de la première moitié de l'année 1922. En février, lord Northcliffe, au retour de son voyage autour du monde, est passé par la Palestine au cours de son itinéraire météorique et perturbateur. Il est dommage que, durant son bref séjour, la maladie ait empêché le haut-commissaire de le recevoir ou de dissiper certaines de ses illusions. Car lord Northcliffe, malade lui-même, est arrivé avec une idée préconçue, acquise pendant son voyage en Extrême-Orient et en Inde, selon laquelle la politique visant à établir un foyer national juif en Palestine était une provocation majeure de l'opinion des musulmans dans le monde et compromettait l'édification de notre empire musulman ${ }^{29}$.

35 À la différence de celle du Daily Express, la couverture de la question palestinienne par le Daily Mail, sera dense, attentive et minutieuse, notamment au cours des années 1920. La brève étape de Northcliffe en Palestine fut, comme il se doit, largement évoquée par le quotidien. Le 9 février 1922, un article à la « une » rendait compte de la rencontre du "Chef » - c'est ainsi qu'on le surnommait parmi son personnel - avec le Grand Mufti de Jérusalem et l'Exécutif sioniste. Le correspondant du journal relate le don de 100 livres sterling fait à l'hôpital St Jean de Jérusalem et surtout quelques extraits significatifs d'un discours prononcé ce jour-là par Northcliffe :

Il a exprimé son regret de constater que la Palestine, qu'il avait connue il y a quelques années, paraissait nettement moins heureuse. Il est convaincu que le public britannique n'est qu'imparfaitement informé des sentiments des Palestiniens. Par exemple [il a déclaré] avoir été approché par d'importantes personnalités juives «orthodoxes» de la communauté ashkénase qui lui ont fait part de leur mécontentement face aux méthodes sionistes actuelles, sur le plan politique et religieux. Et pourtant, [a-t-il poursuivi] le public britannique semble 
croire que tous les Juifs sont cent pour cent sionistes. Lord Northcliffe a conclu en promettant que l'opinion des différentes composantes de la population palestinienne serait accueillie de façon équitable et complète dans ses journaux ${ }^{30}$. manière infléchie, au contraire. En 1923, l'envoyé spécial du quotidien, J.M.N. Jeffries, ancien correspondant de guerre, allait publier une série d'articles - vingt-cinq au total entre le 8 janvier et 8 février sous le titre générique The Deception in Palestine (Palestine : la mystification), détaillant, sous couvert de reportages, les arguments en faveur du retrait britannique du territoire. L'éditorial accompagnant le second volet de la série ( 9 janvier 1923), intitulé «A Real Axe Wanted (Il faut trancher dans le vif), résumait l'argumentaire du quotidien : cette affaire coûte cher au contribuable britannique et ne sert en rien les intérêts du pays. L'argument financier se doublait d'une considération géopolitique. Il était notoire que les dirigeants britanniques redoutaient l'installation en Palestine d'immigrants juifs originaires d'Europe de l'Est suspectés d'avoir des sympathies bolchéviques, ceux que le Daily Mail qualifiait de Judéo-Slaves. La dix-septième livraison du reportage de Jeffries, en date du 26 janvier 1923, était publiée sous le titre suivant : « The Palestine Deception / What Britain has spent / For Slav-Jews to get power ». Le corps de l'article expliquait les raisons de cette prévention:

Les nouveaux groupes d'arrivants en Palestine sont judéo-slaves; leur conception de la vie est judéo-slave ; leur esprit est en proie à une obsession judéo-slave et si cet état de choses se prolonge, une fois passées les premières années de contact avec «l'émancipation» britannique, tout indique que l'on s'oriente vers la constitution d'un État qui, au lieu de protéger l'Égypte, constituera une menace, non seulement pour elle, mais pour le Proche-Orient et les voies de communication britanniques $^{32}$.

Le dernier reportage de Jeffries, en date du 8 février, était annoncé en « une » par la «titraille» suivante: «The Palestine Deception / Zionist claims refuted by J.M.N. Jeffries in concluding article / Mad Finance / Phantom benefits for the Empire but $€ 1,400,000$ in British taxes ». Il se concluait par cette affirmation péremptoire : « La Déclaration Balfour et le Livre blanc [celui de 1922] sont tous deux - et je dis la vérité - malhonnêtes et frauduleux ${ }^{33}$. " L'éditorial du lendemain tirait la conclusion: "M. Jeffries a montré [...] que notre présence en Palestine est basée sur un système sophistiqué de mystification et de malhonnêteté politique dont les factures doivent être honorées par les malheureux Britanniques ${ }^{34}$.» On l'aura compris; l'animosité du Daily Mail à l'égard des sionistes ne se nourrit pas d'un antisémitisme viscéral. Il procède d'une perception précoce de l'impasse dans laquelle se sont engagés les responsables britanniques qui, outre qu'elle s'avère coûteuse, risque de compromettre l'équilibre de l'Empire auquel le quotidien est particulièrement attaché. Du reste, en cohérence avec sa ligne rédactionnelle, le Daily Mail ne limite pas sa demande d'abandon des mandats à la seule Palestine, celui relatif à la Mésopotamie étant également visé par le quotidien. La ligne du Daily Mail sur la question ne variera guère au cours des années suivantes, à ceci près qu'elle perdra de son acuité à mesure que d'autres thèmes de campagnes - celles 
menées conjointement avec Beaverbrook en faveur de la préférence impériale ou de la pacification des dirigeants totalitaires européens - accapareront l'énergie de la rédaction.

Lord Northcliffe disposait d'un autre canal d'expression, autrement plus influent, à savoir le Times, malgré une diffusion six fois moindre que celle du Daily Mail. La notoriété du Times faisait du quotidien un instrument de référence cité aux Communes et une sorte de forum public où s'échangeaient les points de vue dans l'espace important consacré aux lettres à la rédaction (Letters to the Editor). Ainsi, c'est le Times que choisissent D.L. Alexander et Claude Montefiore, respectivement responsable du Conjoint Committee of the Board of Deputies of British Jews et de l'Anglo-Jewish Association, pour publier sous le titre "The Future of the Jews ", le 24 mai 1917, une correspondance dans laquelle ils exprimaient leur opposition à la création d'un État juif autonome qui, selon eux, risquait de mettre à l'épreuve le sentiment d'identité nationale des juifs qui avaient choisi de s'intégrer au pays qui les avait accueillis. Pour les auteurs de la correspondance, le judaïsme était affaire de religion, non de nationalité. Quatre jours plus tard, le Times ouvrait ses colonnes aux réactions de lord Rothschild (Walter Rothschild), du Grand Rabin J.H. Hertz et de Chaïm Weizmann, condamnant l'initiative de ces personnalités qui estimaient pouvoir se mettre en travers de la «concrétisation d'un espoir qui a soutenu la nation juive pendant 2000 ans d'exil, de persécutions et de tentations " rappelle Weizmann ${ }^{35}$. Prenant parti dans la polémique, un éditorial publié le lendemain (29 mai) sous le titre " The Future of the Jews » donnait raison aux trois défenseurs du sionisme, estimant avec eux que ce mouvement concrétisait les aspirations de la plus grande partie de la communauté juive dispersée à travers le monde. L'éditorialiste ajoutait, toutefois, que "l'intérêt $d u$ monde, si l'on excepte la communauté juive, est que ces aspirations, pour autant qu'elles soient susceptibles de se traduire concrètement, puissent être examinées de façon impartiale en fonction de leurs mérites ${ }^{36}$.»

40 La position du Times allait nettement évoluer après la Déclaration Balfour et surtout à la suite du bref séjour de Northcliffe en Palestine déjà évoqué. Le quotidien publiera à cette occasion un commentaire très réservé de celui qui commence par réaffirmer son soutien initial au projet sioniste "qui nous semblait offrir l'occasion de sortir les juifs de la position ambiguë et anormale qui est la leur dans de nombreux pays et paraissait de nature à leur offrir la possibilité de retrouver un équilibre naturel grâce à l'affirmation progressive de leur spécificité nationale ${ }^{37} »$. Northcliffe mentionne deux objections au projet sioniste tel qu'il le voit se développer. D'abord, tous les Juifs ne se réclament pas du sionisme ; beaucoup inscrivent leur judaïté dans la sphère religieuse et non dans une revendication nationale. Ensuite, estime Northcliffe, le projet risque d'être compromis par l'arrivée de Juifs d'Europe de l'Est, la tête pleine « d'espoirs démesurés et de demandes irréalistes et qui n'ont aucune idée de la complexité de la situation ${ }^{38}$. $\gg$ Dans le même texte, Northcliffe réclamait, comme il l'avait fait dans les colonnes du Daily Mail, la mise en place d'une enquête officielle sur la question palestinienne, demande à laquelle souscrivait le lendemain Chaïm Weizmann dans une lettre à la rédaction.

41 Northcliffe avait laissé en Palestine un correspondant du Times, Philip Graves, qui en mars et en avril 1922 publiera dans les colonnes du quotidien une série de reportages dont un éditorial, le 11 avril, tentera la synthèse. Dès le premier paragraphe apparait la réserve majeure - le contribuable britannique n'a pas été consulté -, réserve bientôt élargie à la remise en cause globale de la Déclaration Balfour : « Rétrospectivement, il parait incroyable que les responsabilités impliquées par la Déclaration Balfour aient été souscrites si légèrement, avec si peu de capacité d'anticipation et avec une telle absence de perspective 


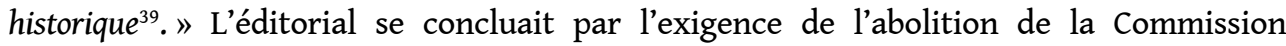
sioniste accusée de jouir de privilèges excessifs et suspectée de desseins politiques inavoués. Allaient suivre d'autres éditoriaux de la même veine, tel celui du 26 avril 1922 intitulé «The Burden of Palestine » réclamant à nouveau la suppression de la commission soupçonnée de s'arroger des pouvoirs indus en matière de gestion de l'immigration.

La tonalité des articles du Times traitant de la question palestinienne perdra son aspérité après la mort de Northcliffe (août 1922), sans que, pour autant, les interrogations essentielles, touchant la capacité du gouvernement à résoudre les engagements contradictoires impliqués par la Déclaration Balfour et surtout le coût de cette politique, disparaissent du champ des questionnements des éditorialistes. Si, au lendemain de la publication du Livre blanc de lord Passfield, le Times estime que la politique poursuivie depuis la conquête du territoire en 1917 est la seule capable de servir les intérêts légitimes de la population arabe et ceux, non moins légitimes, des sionistes, l'éditorialiste s'inquiète de l'augmentation des dépenses nécessaires au fonctionnement d'une administration palestinienne pléthorique et à l'entretien d'une garnison britannique constamment renforcée, sans perspective discernable de retrait (21 octobre 1930). Deux jours plus tard, c'est dans le Times que les chefs de file du parti conservateur, alors dans l'opposition,- Stanley Baldwin, Austen Chamberlain et Leo Amery- choisissaient d'affirmer leur rejet des conclusions du Livre blanc, jugées en contradiction, non seulement avec les termes du mandat, mais aussi avec la politique menée depuis douze ans par les gouvernements britanniques successifs. Le 4 novembre, c'était au tour de lord Hailsham et de John Simon de contester les points du Livre blanc concernant les restrictions d'accès des juifs aux terres encore disponibles et la limitation des flux d'immigration. Deux jours plus tard, lord Passfield tentait de démontrer que les " déductions opérées par lord Hailsham et Sir John Simon sont infondées et reposent sur une interprétation erronée des intentions officielles du gouvernement de Sa Majestét ${ }^{40}$. " La polémique s'est poursuivie jusqu'à la fin de l'année 1930, chaque nouvelle lettre entraînant un correctif ou une nouvelle mise au point, quand il ne s'agissait pas de prises de position péremptoires comme celle du colonel Meinertzhagen. L'ancien collaborateur de Churchill au Colonial Office concluait sa lettre au Times, publiée le 21 novembre, par la profession de foi suivante : "Le sionisme est aujourd'hui une réalité établie pour longtemps. Tenter de faire obstacle à la communauté juive revient à faire obstacle à l'histoire. Le gouvernement de Sa Majesté et les ennemis du sionisme peuvent retarder l'évolution de la Palestine vers son destin ultime, mais ils ne peuvent en empêcher l'accomplissement ${ }^{41}$. "

43 La montée de l'activisme arabe au cours des années trente, empruntant de plus en plus la voie de la violence terroriste, ne pouvait que conforter le Times dans l'idée que le pays s'épuisait à tenir des engagements irréalistes, parce que contradictoires, rendus encore plus inaccessibles par l'exacerbation de l'antagonisme qui opposait les deux communautés. C'est également l'analyse du Daily Herald au lendemain de la publication du rapport Peel (juillet 1937) préconisant le partage de la Palestine entre deux États souverains et une zone placée sous mandat. "Nous avons vendu le même cheval, qui ne nous appartenait pas, à deux acheteurs différents » résumait l'éditorial du 8 juillet 1937 publié sous le titre purement référentiel de $"$ Palestine $»^{42}$. L'éditorialiste estimait, par ailleurs, que les propositions de la Commission Peel, dont on savait par avance qu'elles seraient mal accueillies, étaient, à tout prendre, celles qui étaient le plus susceptibles de régler un problème pratiquement insoluble - « a well-nigh insoluble problem ». 

rapport Peel. Proche du parti travailliste, le quotidien n'est pas insensible aux arguments de Morgan Jones, porte-parole du Labour sur la question, qui, aux Communes, juge le plan de partition insensé et inacceptable. Le projet de « corridor » reliant Jérusalem au port d'Haïfa, placé sous mandat britannique, lui paraît particulièrement irréaliste : «J'aurais cru que les membres de cette Chambre avaient eu leur content de 'corridors'. La situation en Prusse orientale vous satisfait-elle tant que vous vouliez un corridor supplémentaire?» déclarait le parlementaire aux Communes $^{43}$, avant de réclamer l'avis d'une commission mixte composée de représentants des Communes et de la Chambre des lords préalablement à la soumission du rapport Peel au Parlement pour approbation. Rapportés en détail, les propos du député travailliste étaient prolongés par un éditorial dans lequel la rédaction appuyait la demande d'examen préalable par une commission mixte après avoir fait part de ses doutes: «La partition apparait aux membres de la Commission [Peel], après une étude approfondie de la question, comme la seule solution possible. Mais, plus on examine leurs propositions et moins il semble qu'elles puissent en fait être appliquées, aussi ingénieuses soientelles ${ }^{44}$."

Le Daily Herald restera constamment attentif à la réaction des différents protagonistes, notamment à celle des sionistes dont le congrès mondial, réuni à Zürich en août 1937, amènera Chaïm Weizmann à revenir sur sa position initialement favorable à la constitution d'un État juif dans les limites territoriales préconisées par le rapport Peel. La question palestinienne était suivie au Daily Herald par A.L.Easterman qui était particulièrement bien informé, au point d'annoncer bien avant la publication du rapport Peel l'essentiel de ses recommandations, ce dont le quotidien tire quelque fierté dans un encadré publié dans l'édition du 5 juillet : «Les recommandations de la Commission sont très largement conformes aux prévisions publiées par le Daily Herald le 2 avril et le 17 juin ». Les informations livrées en avant-première par le Herald eurent pour effet de déclencher un flot de réactions, tant en Europe qu'aux États-Unis, dont beaucoup furent transmises au Colonial Office. Les plus pertinentes furent vraisemblablement prises en compte par la Commission Peel pour modifier en conséquence le tracé des zones assignées aux futurs États en gestation ${ }^{45}$.

\section{Conclusion}

Pour importante qu'elle puisse paraître rétrospectivement, la question palestinienne ne semble pas avoir retenu l'attention soutenue de la presse britannique tout au long de la période. D'autres enjeux ont monopolisé l'actualité au cours de l'Entre-deux-guerres, notamment la question irlandaise, la crise financière de 1929, le règlement du problème indien et la montée en puissance des régimes totalitaires en Europe. Certains titres ont été mis au service des positions hostiles au sionisme de leur propriétaire - lord Northcliffe en particulier -, d'autres ont connu une évolution de leur ligne rédactionnelle en fonction de l'enlisement des positions sur le terrain et à mesure que se confirmait l'incapacité des autorités britanniques à mettre en place des solutions viables. Tel fut notamment le cas du Times qui servit tout au long de la période de caisse de résonance à la question palestinienne, en faisant office de forum et de cadre d'échanges entre les différents protagonistes. Ce sera également le rôle que s'efforcera de jouer au cours des années trente le Daily Herald, ancré à gauche et donc, par implication, plus ouvert à la

Revue Française de Civilisation Britannique, XVII-2 | 2012 
cause sioniste, encore que celle-ci ait très largement transcendé les clivages partisans de l'époque.

Il ne semble pas non plus que la presse ait collectivement été en mesure d'influer sur les décisions, sauf peut-être, de façon marginale, en ce qui concerne la préparation du Livre blanc de 1922 réclamé par Northcliffe. La presse a essentiellement joué un rôle réactif, dont les manifestations ont contribué à établir un climat dont les politiques ont nécessairement tenu compte, sans qu'il soit possible d'en mesurer précisément le poids dans l'élaboration des différents plans de règlement qui se sont succédés au cours de la période.

\section{NOTES}

1. «[...] my own authority and that of every department of my Administration is claimed or impinged upon by the Zionist Commission and I am definitely of opinion that this state of affairs cannot continue without grave danger to the public peace", rapporté par The Daily Mail, 17 janvier 1923.

2. "Under the stress of the World War the British Government made promises to Arabs and Jews in order to obtain their support. On the strength of these promises both parties formed certain expectations [...]. An irrrepressible conflict has arisen between two national communities within the narrow bounds of the small country [...]. The conflict was inherent in the situation from the outset. ». Palestine - Royal Commission Report (The Peel report), Cmd 5479, Londres : HMSO, 1937, p. 370.

3. "The old account with the Jewish people had to be squared if the new world was to start with a clean balance-sheet. " Chaïm Weizmann, allocution au dix-septième Congrès sioniste, 1 juillet 1931 in Barnet LITVINOFF (dir.), The Letters and Papers of Chaim Weizmann, vol. I, série B, Rutgers University and Israel University Press, Transaction Books, 1983, p. 614.

4. Voir Leonard STEIN, The Balfour Declaration, Londres : Valentine, Mitchell, 1961, pp. 103-104.

5. Il s'agit de Walter Rothschild, fils aîné de lord Leopold Rothschild, décédé en 1917, lequel ne se rallia que sur le tard au projet sioniste auquel n'adhérait pas son plus jeune fils Lionel.

6. Correspondence with the Palestine Arab Delegation and the Zionist Organisation. And a Statement of British Policy in Palestine (The Churchill White Paper), Cmd. 1700, Londres : HMSO, juin 1922.

7. "I cannot exclude from my mind the possibilty of further disturbances or even, as my military advisers have warned me, of a general uprising. " Lettre de Samuel à Churchill, 13 juin 1921. Rapporté par Bernard WASSERSTEIN, The British in Palestine - The Mandatory Government and the Arab-Jewish

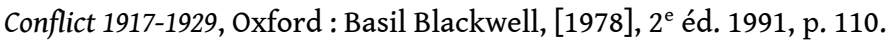

8. "The British Government, the trustee under the Mandate for the happiness of the people of Palestine, would never impose upon them a policy which that people had reason to think was contrary to their religious, their political and their economic interests. " Rapporté par Bernard WASSERSTEIN, Ibid., p. 110.

9. En date du 24 octobre 1915, Sir Henry McMahon, haut-commissaire britannique en Égypte, adressait à Hussein, chérif de la Mecque, une lettre lui garantissant, en échange du soulèvement des Arabes contre les Turcs, le soutien du Royaume-Uni à la création d'un État arabe indépendant aux frontières imprécises. Les leaders arabes affirmeront que la Palestine faisait partie de cet État virtuel, ce que contesteront inlassablement les dirigeants britanniques. Le texte de la lettre restera longtemps secret. 
10. Palestine - Statement of Policy by His Majesty's Government in the United Kingdom Presented by the Secretary of State for the Colonies to Parliament, Cmd. 3692, Londres: HMSO, octobre 1930.

11. "If immigration of Jews results in preventing the Arab population from obtaining the work necessary for its maintenance, or if Jewish unemployment unfavourably affects the general labour position, it is the duty of the Mandatory Power under the Mandate to reduce or, if necessary, to suspend, such immigration until the unemployed portion of the 'other sections' is in position to obtain work. » Ibid., par. 28.

12. "The Passfield White Paper was considered to be formulated in a manner calculated to cause offence. That, at any rate, is how the Jews reacted. And Beatrice Webb, who could by no standard be considered sympathetic towards Zionism [...] confirms this when she recorded that 'The Statement [...] is a badly drafted, a tactless document'. » N.A. Rose, The Gentile Zionists - A Study in Anglo-Zionist Diplomacy 1929-1939, Londres : Frank CASS, 1973, p. 17.

13. "In order to remove certain misconceptions and misunderstandings which have arisen as to the policy of His Mjesty's Government with respect to Palestine, as set forth in the White Paper of October, 1930, and which were the subject of a debate in the House of Commons on November 17, and also to meet certain criticisms put forward by the Jewish Agency, I have pleasure in forwarding you the following statement of our position, which fall to be read as the authoritative interpretation of the White Paper on the matters with which this letter deals. » The Times, 15 février 1931.

14. "[...] it twas under MacDonald's letter to me that the change came about in the Government's attitude and in the attitude of the Palestine administration which enabled us to make the magnificient gains of the ensuing years. " Chaïm Weizmann, Trial and Error, 1950. Rapporté par Charles L. GEEDES (dir.), A Documentary History of the Arab-Israeli Conflict, New York : Praeger, 1991, p. 152.

15. Palestine Royal Commission Report (The Peel Report), Cmd. 5479, Londres : HMSO, 1937.

16. "This report provides by far the most comprehensive and wide-ranging analysis of the situation in Palestine to be produced by a British authority. " Penny Sinanoglou, "The Peel Commission and Partition, 1936-1938" in Rory MILLER (dir.), Britain, Palestine and Empire - The Mandate Years, Londres: Ashgate, 2010, p. 119.

17. "The political, administrative and financial difficulties involved in the proposal to create independent Arab and Jewish States in Palestine are so great that this solution is impracticable. " Palestine. Statement by His Majesty's Government in the United Kingdom, Cmd. 5893, Londres: HMSO, novembre 1938. Rapporté par Charles L. GEDDES, op. cit., p. 184.

18. Palestine: Statement of Policy, Cmd. 6019, Londres : HMSO, 1939.

19. "It is a policy which [...] sets up a territorial ghetto for the Jews in their homeland.» Rapporté par Charles L. GEDDES, op. cit., p. 198.

20. "[He said that] the average newspaper reader thought that there was a Jewish State in Palestine, meaning by 'State' what was usually meant in Prussia, a sort of body which rode roughshod over anybody who did not happen to belong to the ruling authority. This State was maintained (it was said) by British bounty, and was paid by British taxes. That was, more or less, the opinion which prevailed in the mind of the average newspaper reader .» Discours prononcé à Oxford le 22 février à l'invitation de la Société sioniste de l'Université, in Barnet LITVINOFF (dir.), The Letters and Papers of Chaim Weizmann, vol. I, op. cit., p. 341.

21. "Agitation in the British Press at this time accused Zionism, and Weizmann specifically, of organizing Britain's entanglement with Palestine, to the cost of the British taxpayer.» Ibid., note 67, p. 341.

22. "A document in which the British Government would express its sympathy with the Zionist movement and its intention to support the creation of a Jewish national home in Palestine. » Rapporté par David AYERST, Guardian - Biography of a Newspaper, Londres : Collins, 1971, p. 385.

23. "We speak of Palestine as a country, but it is not a country; it is at present little more than a small district of the vast Ottoman tyranny. But it will be a country, it will be the country of the Jews." Manchester Guardian, 5 novembre 1917.

24. « Palestine must either be part of Egypt [...], or it must be a buffer State which is prevented from becoming hostile to Egypt. On the realisation of that condition depends the whole future of 
the British Empire as a sea Empire. » Rapporté par Herbert Sidebotham, Great Britain and Palestine, Londres : Macmillan and Co, 1937, p. 27.

25. «In 1918 the anti-zionist Beaverbrook deprecated Zionist propaganda on the ground that the Ministry of Information had no success with religious material. » Jason Tomes, Balfour and Foreign Policy, Cambridge University Press, 1997, p. 200.

26. «I have been for years a prophet of no war. But at last I am shaken. The Jews may drive us into war; I do not mean with any conscious purpose of doing so. They do not mean to do it. But unconsciously they are drawing us into war; their political inflence is moving us in that direction. » Rapporté par A.J.P. Taylor, Beaverbrook, New York : Simon \& Schuster, 1972, p. 387.

27. "The Jews have got a big position in the press here. I estimate that one-third of the circulation of the Daily Telegraph is Jewish. The Daily Mirror may be owned by Jews. The Daily Herald is owned by Jews. And the News Chronicle should really be the Jewish Chronicle. Not because of ownership but because of sympathy. The Times, the Daily Mail and the Express are the only papers left. And I am not sure of the Mail.» Ibid.

28. "The policy of His Majesty's Government in Palestine is that laid down by the Foreign Secretary in his last speech. Until it is altered officially, it is in no way affected by conversations between Sir Charles Henry and the Prime Minister. » Lord BEAVERBROOK, Men and Power 1917-1918, Londres : Hutchinson, 1956, pp. 292-293.

29. "The Press and parliamentary campaign of misrepresentation reached its peak during the first half of the year 1922. In February of that year Lord Northcliffe, returning from his tour round the world, passed through Palestine in his meteoric and disturbing course. It was unfortunate that, during his short visit, the High Commissioner was seriously ill to see his guest or remove any of his illusions. For Lord Northcliffe, who was ill himself, came with a prepossession gathered during his journey in the Far East and India that the policy of the Jewish National Home in Palestine was a major provocation of Moslem opinion throughout the world and endangered the foundations of our Moslem Empire.", Norman BENTWICH, England in Palestine, Londres : Kegan Paul, Trench, Trubner \& Co, 1932, p. 79.

30. " He expressed his regret that Palestine, which he had known years ago, was now apparently decidedly less happy. He believed that the British public is imperfectly informed in regard to Palestinian feeling. For example, he had been approached by prominent 'othodox' Jews of the Ashkenazim who expressed profound discontent with the present Zionist methods from a political and religious standpoint. Yet the British public appeared to believe that every Jew is an out and out Zionist. Lord Nothcliffe [...] concluded with a promise that the opinions of all sections of Palestians would have a fair and full hearing in his newspapers. "The Daily Mail, 9 février 1922.

31. "In my opinion, unless the situation is firmly dealt with and greater respect is shown for the right of 700,000 Palestinian Moslems and Christians, the country runs the risk of becoming a second Ireland. " The Daily Mail, 16 février 1922.

32. "The new groupings in Palestine are Judeo-Slav; their attitude upon life is Judeo-Slav; their mental intoxication is Judeo-Slav; and should the present régime continue, when the first few years in contact with Britannic 'emancipation' have been passed, all points to the constitution of a State not protective but perilous to Egypt, to the Near East, and to British communications. " The Daily Mail, 26 janvier 1923.

33. "The Balfour Declaration and the White Book are both-and I speak the truth-dishonest and fraudulent. " The Daily Mail, 8 février 1923.

34. «Mr. Jeffries has shown [...] that our presence in Palestine is based on an elaborate system of deception and political fraud for which the unfortunate British public have to pay the bills. » Éditorial du Daily Mail du 9 février 1923 publié sous le titre « Evacuate the Near East».

35. "The realization of a hope which has sustained the Jewish nation through 2,000 years of exile, persecution and temptation. » Chaïm Weizmann in The Times, 28 mai 1917.

36. " The interest of the world outside Jewry is that these aspirations, in so ar as they may be susceptible of realization, should be fairly faced on their merits. » The Times, 29 mai 1917. 
37. "[...] which seemed to us to afford an opportunity of releasing the Jews from the ambiguous and anomalous position that they occupy in many countries, and of enabling them to recover a natural equilibrium by a progressive affirmation of their national individuality." The Times, 17 février 1922.

38. "They come to the home of their remote ancestors [...] with extravangant hopes and impossible claims and have no perception of very complex realities. » Ibid.

39. "As we look back now it appears incredible that the responsibilities involved in the Balfour Declaration should have been undertaken so lightly, with so little forethought, and with such a lack of historical perspective. " The Times, 11 avril 1922.

40. "[...] the inferences drawn by Lord Hailsham and Sir John Simon are unfounded and are based upon a misconception of the declared intentions of His Majesty's Government. » The Times, 6 novembre 1930.

41. "Zionism has come to stay. To attempt to interfere with Jewry is to interfere with history. His Majesty's Government and enemies of Zionism can delay the ultimate destiny of Palestine but they cannot prevent its ultimate fulfilment. » The Times, 21 novembre 1930.

42. "We sold a horse that wasn't ours to two different buyers ». The Daily Herald, 8 juillet 1937.

43. "I should have thought [...] that most members of this House would have enough by now of 'corridors'. Do you like the situation in East Prussia so much that you want another corridor?" The Daily Herald, 22 juillet 1937.

44. "Partition seems to the Commissionners, after an intensive study of the question, the only possible solution. But the more their proposals are examined, the less likely does it seem that, however ingenious, they should work in fact. » Ibid.

45. Voir sur ce point Penny SINANOGLOU, « The Peel Commission and Partition, 1936-1937 » in Rory MILLER, Britain, Palestine and Empire: The Mandate Years, op. cit., pp. 128-132.

\section{RÉSUMÉS}

Les gouvernements britanniques qui se sont succédé au pouvoir au cours de l'Entre-deux guerres se sont invariablement heurtés à l'insoluble contradiction en germe dans la Déclaration Balfour. De Livres blancs en rapports de commissions, la position des responsables britanniques évoluera vers une plus grande prise en compte des réalités palestiniennes, avant que la Deuxième Guerre mondiale n'impose d'autres priorités.

La question palestinienne est globalement peu traitée par la presse britannique, à l'exception de certains titres dont les propriétaires ont défendu leur vision personnelle de l'engagement britannique en Palestine, jugé coûteux et voué à l'échec. Telle était notamment la position de lord Northcliffe, largement développée dans le Daily Mail et, dans une moindre mesure, dans le Times qui a essentiellement servi de tribune aux différents protagonistes

The successive British governments which alternated in office during the interwar years invariably failed to solve the contradictory commitments included in the Balfour Declaration. The British official stance as reflected in the numerous White Papers and reports devoted to the issue gradually evolved towards a more realistic approach to the Palestinian question which was sidelined with the outbreak of World War Two.

Palestine did not get much in-depth coverage by the British Press except in those dailies owned by opinionated newspaper proprietors such as Lord Northcliffe who used The Daily Mail to criticize the British Mandate as both too costly and doomed to fail. The Times also aired Lord 
Northcliffe's views but was mostly used as a tribune by all those involved in the handling of the Palestinian question.

\section{AUTEUR}

\section{JEAN-CLAUDE SERGEANT}

Jean-Claude Sergeant est professeur émérite à l'université de la Sorbonne Nouvelle-Paris 3). Spécialiste de civilisation britannique, il a publié, seul ou en collaboration, une demi-douzaine d'ouvrages et une centaine d'articles consacrés aux médias et à la vie politique britanniques. 